\title{
Aplicação da Equação do Calor em Coordenadas Cilíndricas: Cálculo da Difusividade Térmica
}

\author{
Mônica F. de Sousa, José S. Sales \& Orlene S. da Costa
}

Neste trabalho, apresenta-se um método para a obtenção da difusividade térmica de um creme hidratante imerso num recipiente cilíndrico metálico com termopares colocados nas posições radiais. Para isso, foi resolvida a equação do calor em coordenadas cilíndricas, sem fonte de calor interno, considerando a propagação do calor radialmente no meio isotrópico e homogêneo, obtendo uma expressão analítica completa.

Palavras-chave: Difusividade térmica; creme hidratante; equação do calor.

In this paper we present a method for obtaining the thermal diffusivity of a cream moisturizer immersed in a metal cylindrical container with thermocouples placed at radial positions. For this we solve the heat equation in cylindrical coordinates without internal heat source, considering the propagation of heat radially in middle isotropic and homogeneous, obtaining a complete analytical expression.

Keywords: thermal diffusivity; cream moisturizing; the heat equation. 


\section{Introdução}

As propriedades físico-químicas são importantes nos estudos de sólidos e líquidos que sofrem aquecimento e resfriamentos. A difusividade térmica é uma dessas propriedades que indica a velocidade com que o calor se propaga através da substância, permitindo conhecer como alguns parâmetros físico-químicos são alterados ${ }^{1}$.

A difusividade térmica também é conhecida como a razão entre a condutividade térmica ke o produto da capacidade térmica $c_{p}$ e a massa específica $\rho$, que mede a razão entre a capacidade da substância conduzir energia e a capacidade de acumular calor dada por ${ }^{2}$ :

$$
\alpha=\frac{k}{\rho \cdot c_{p}}
$$

Muitos métodos são classicamente utilizados para determinação da difusividade térmica de substâncias sólidas, líquidas e pastosas, por exemplo, por meio da razão entre condutividade térmica e o produto da massa específica e calor específico reportado no artigo de $\mathrm{SWEAT}^{3}$, da fonte de calor secundária de calor, em forma de bastão acoplado a um termopar inserido no sólido e fixado em paralelo a fonte de calor principal ${ }^{4}$ e de observação da distribuição da temperatura no interior da amostra ou fluido durante o aquecimento ou resfriamento ${ }^{5}$. Citando ainda o modelo condutivo associado a um algoritmo de otimização, que calcula a soma dos quadrados residuais obtidos da comparação entre os dados experimentais e simulados ${ }^{6}$. Na literatura também aparece determinação da difusividade térmica de alimentos com soluções analíticas com o uso da equação do calor, por exemplo, no artigo de Markowski ${ }^{7}$ e no artigo de Oro ${ }^{8}$.

A equação da propagação do calor $\mathrm{U}=\mathrm{U}(\mathrm{r}, \varnothing, \mathrm{z}, \mathrm{t}) \mathrm{em}$ coordenadas cilíndricas é dada por ${ }^{9}$ :

$$
\frac{1}{r} \cdot \frac{\partial}{\partial r}\left(r \frac{\partial U}{\partial r}\right)+\frac{1}{r^{2}} \cdot \frac{\partial^{2} U}{\partial \emptyset^{2}}+\frac{\partial^{2} U}{\partial z^{2}}+\frac{\dot{q}}{K}=\frac{1}{\alpha} \cdot \frac{\partial U}{\partial t},
$$

\section{Detalhes Experimentais}

Para o método, foi contruído artesanalmente um recipiente cilíndrico, metálico, com diâmetro de 18,30 $\mathrm{cm}$, altura de $5,80 \mathrm{~cm}$ e espessura da parede de $0,10 \mathrm{~cm}$, isolado termicamente no topo e na base, contendo creme hidratante corporal, a fim de promover a transferência de calor radialmente, regime transiente, visto na Figura 1.

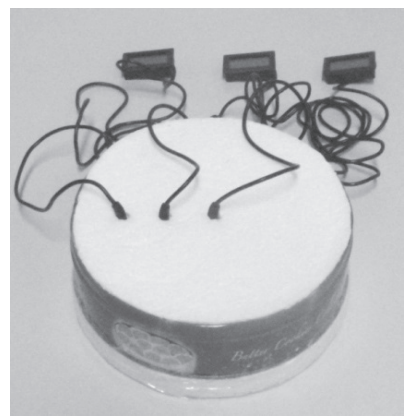

Figura 1. Recipiente metálico contendo creme hidratante corporal, isolado termicamente na base e no topo com isopor na tampa dotada de três termopares digitais na direção radial.

O conjunto metálico, creme hidratante corporal, foi colocado em um banho termostatizado, com temperatura de $40^{\circ} \mathrm{C}$, mostrado na Figura 2 .

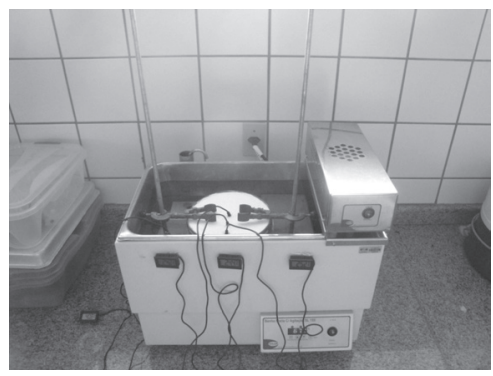

Figura 2. Sistema cilíndrico de transferência radial de calor por condução, inserido em banho termostatizado, com termopares acoplados.

O binômio, tempo e temperatura, foi registrado durante 90 minutos, com intervalo de dois em dois minutos. As temperaturas foram registradas nas posições $6,10 \mathrm{~cm}, 3,05 \mathrm{~cm}$ e $0,00 \mathrm{~cm}$, respectivamente, de acordo com a distribuição da temperatura mostrada na Figura 5.

sendo $q^{\prime}$ a fonte de calor interna. 


\section{ANÁLISE MATEMÁTICA DA TRANSFERÊNCIA DE CALOR COM CONDIÇÕES DE CONTORNO}

Nesta seção, será apresentada a solução da equação do calor com as condições de contorno, de acordo com as medidas experimentais descritas, a fim de obter e apresentar um método simples para o cálculo da difusividade térmica, com a propagação do calor ocorrendo radialmente.

Considerando a substância isotrópica e homogênea, sem fonte interna de calor, e com a propagação do calor ocorrendo radialmente a equação (2), torne-se:

$$
\frac{1}{r} \cdot \frac{\partial}{\partial r}\left(r \frac{\partial U}{\partial r}\right)=\frac{1}{\alpha} \cdot \frac{\partial U}{\partial t},
$$

Agora a distribuição de temperatura $\mathrm{U}(\mathrm{r}, \varnothing, \mathrm{z}, \mathrm{t})=$ $\mathrm{U}(\mathrm{r}, \mathrm{t})$, usando o método de separação de variáveis com $\mathrm{U}(\mathrm{r}, \mathrm{t})=\mathrm{R}(\mathrm{r}) \mathrm{T}(\mathrm{t})$, têm-se duas equações independentes dada por:

$$
\frac{1}{\alpha T(t)} \frac{\partial T(t)}{\partial t}=-\lambda^{2},
$$

cuja a solução é dada por:

$$
T(t)=A+B e^{-\lambda^{2} \alpha t},
$$

e a outra é dada por:

$$
r^{2} \frac{\partial^{2} R(r)}{\partial r^{2}}+r \frac{\partial R(r)}{\partial r}+\lambda^{2} r^{2} R(r)=0,
$$

sendo a solução em termo da série de Bessel ${ }^{10}$ dada por:

$$
R(r)=J_{0}(\lambda r)=\sum_{n=0}^{\infty} \frac{(-1)^{n}}{n ! \Gamma(n+1)}\left(\frac{\lambda r}{2}\right)^{2 n},
$$

cujo gráfico da função de Bessel $\mathrm{J}_{0}(\mathrm{x})$ é mostrado na Figura 3.

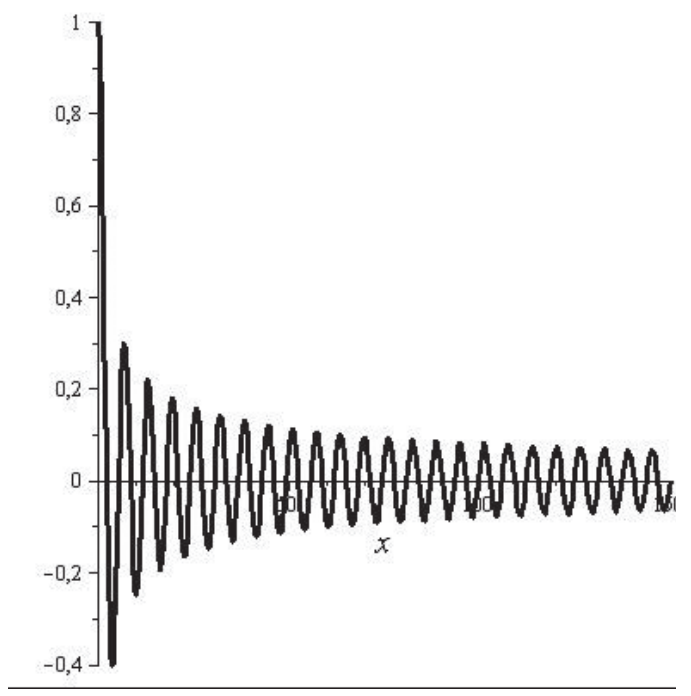

Figura 3. Gráfico da função de Bessel $\mathrm{J}_{0}(\mathrm{x})$.

Generalizando, ou seja, fazendo $\lambda \mathrm{r} \rightarrow \lambda_{\mathrm{i}}$ ra solução geral da equação (3) pode ser escrita por:

$$
U(r, t)=C_{1}+\sum_{i=0}^{\infty} C_{i} e^{-\lambda_{i}^{2} \alpha t} J_{0}\left(\lambda_{i} r\right),
$$

sendo que os coeficientes $\mathrm{C}_{1}$ e $\mathrm{C}_{\mathrm{i}}$ foram determinados usando a condição que $\mathrm{t} \rightarrow \infty$ a temperatura da substância é a temperatura do banho $T_{f}$, ou seja,

$$
U(r, \infty)=C_{1}=T_{f}
$$




\section{Artigo Geral 2}

Mas, na condição em que a medida da temperatura na extremidade $r=a$, raio do recipiente, é também a temperatura do banho $T_{f}$, para qualquer $t$, inclusive em $\mathrm{t}=0$ tem-se:

$$
U(a, t)=T_{f},
$$

basta encontrar graficamente $\lambda_{i} r=x \_i$, na Figura 3, as raízes da função de Bessel $\mathrm{J}_{0}\left(\lambda_{\mathrm{i}} \mathrm{a}\right)$. E no instante inicial $\mathrm{t}=0$, para qualquer posição $\mathrm{r}$, tem-se que a temperatura do material é a temperatura ambiente $\mathrm{T}_{\mathrm{m}}$, dada por:

$$
T_{m}-T_{f}=\sum_{i=0}^{\infty} C_{i} J_{0}\left(\lambda_{i} r\right)
$$

usando a ortogonalidade das funções Bessel ${ }^{11,12}$, os coeficientes podem ser calculados por:

$$
C_{i}=\frac{\left(T_{m}-T_{f}\right) \int_{0}^{a} r J_{0}\left(\lambda_{i} r\right) d r}{\int_{0}^{a} r J_{0}^{2}\left(\lambda_{i} r\right) d r} .
$$

Das relações de recorrência das funções de Bessel tem-se:

$$
C_{i}=\frac{2\left(T_{m}-T_{f}\right)}{\lambda_{i} a J_{1}\left(\lambda_{i} a\right)},
$$

portanto, a solução da equação do calor na distribuição da temperatura com a propagação radial do calor em substâncias homogêneas e isotrópicas, será dada por:

$$
U(r, t)=T_{f}+2\left(T_{m}-T_{f}\right) \sum_{i=0}^{\infty} \frac{e^{-\lambda_{i}^{2} \alpha t}}{x_{i} J_{1}\left(\lambda_{i} a\right)} J_{0}\left(\lambda_{i} r\right),
$$

sendo

$$
J_{1}(\lambda r)=\sum_{n=0}^{\infty} \frac{(-1)^{n}}{n ! \Gamma(n+2)}\left(\frac{\lambda r}{2}\right) \cdot{ }^{2 n+1}
$$

Para a posição $\mathrm{r}=6,10 \mathrm{~cm}$, nos instantes $16 \mathrm{~min}$ (sólida), $30 \mathrm{~min}$ (pontilhada), $40 \mathrm{~min}$ (tracejada) e 70 min (traço-ponto), traça-se o gráfico da distribuição da temperatura $U(r, t)$ em função da difusividade $\alpha$, Figura 4 . Neste gráfico, com o auxílio do gráfico da distribuição de temperatura, da Figura 5, com as respectivas temperaturas, encontra-se a difusividade térmica média $\alpha_{\mathrm{m}}=1.49 \times 10^{-5}$ $\mathrm{m}^{2} / \mathrm{min}=2.48 \times 10^{-7} \mathrm{~m}^{2} /$ sdo creme hidratante corporal.

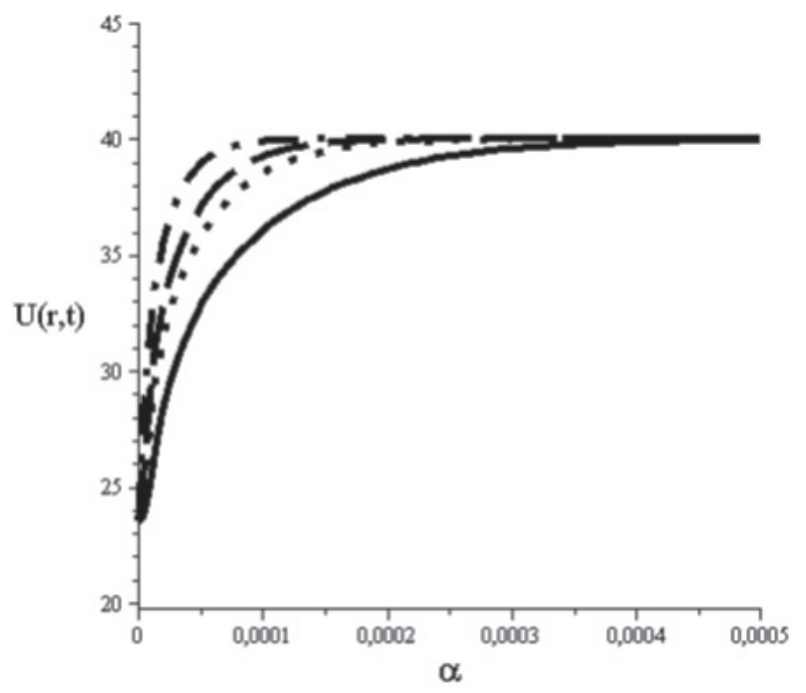

Figura 4. Distribuição da temperatura em função da difusividade para $\mathrm{r}=6,10 \mathrm{~cm}$ nos instantes $16 \mathrm{~min}$ (sólida), $30 \mathrm{~min}$ (pontilhada), $40 \mathrm{~min}$ (tracejada) e 70 min (traço-ponto).

Para as posições $r=3.10 \mathrm{~cm}$ e $r=0,0 \mathrm{~cm}$, em vários instantes, simulados os gráficos, apresentaram o mesmo comportamento.

No gráfico mostrado na Figura 5, apresenta-se também o resultado teórico (curvas sólidas), a partir da solução $U(r, t)$ da equação do calor, dada pela Equação (15), para a posição $\mathrm{r}=6.10 \mathrm{~cm}$ (asterisco), $\mathrm{r}=3,05$ (cruz) $\mathrm{cm}$ e $\mathrm{r}=0,0 \mathrm{~cm}$ (quadrada). 


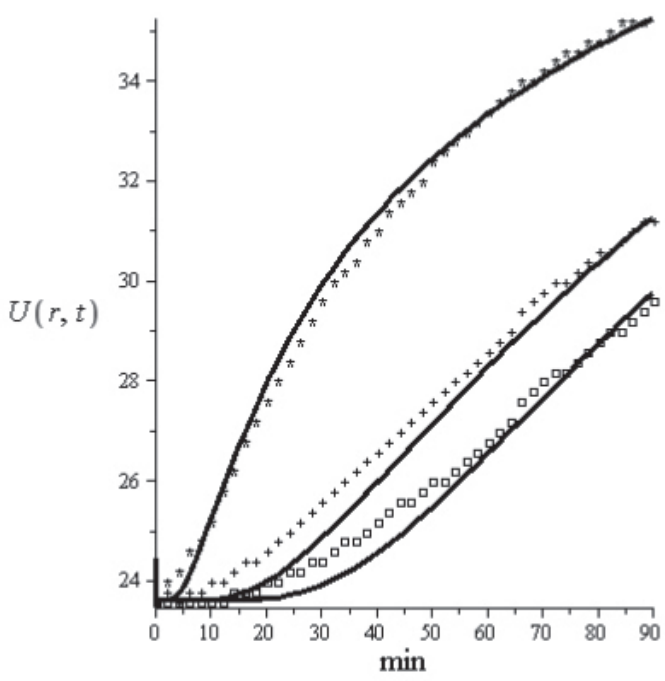

Figura 5. Distribuição da temperatura em função do tempo para $r=$ $6,10 \mathrm{~cm}$ (asterisco), $\mathrm{r}=3,05 \mathrm{~cm}$ (cruz), $\mathrm{r}=0$ (quadrada).

Conforme esperado, a difusividade térmica do creme hidratante corporal é menor que, por exemplo, a difusividade térmica do cobre, dada por $\alpha=1153 \times 10^{-7}$ $\mathrm{m}^{2} / \mathrm{s}$, uma vez que o cobre é um melhor condutor de calor do que o creme hidratante.

\section{Conclusão}

A grande vantagem do método apresentado é a sua simplicidade de realização experimental. Medindo a temperatura em um dado instante, numa determinada posição radial da substância, colocada num cilindro imerso num banho termostatizado, considerando a substância homogênea e isotrópica, e com o auxílio da Equação (15), encontrou-se a difusividade térmica diretamente no gráfico da temperatura em função difusividade térmica. Neste, foi encontrado um valor médio da difusividade térmica de um creme hidratante corporal menor do que a difusividade térmica do cobre, como esperado, pois se considerada a mesma área e a mesma espessura, uma parede do creme aquecido, este se aquecerá mais lentamente do que o cobre na mesma condição. O desvio das curvas das distribuições das temperaturas experimentais com as curvas teóricas, na Figura 5, classicamente, é, com certeza, devido aos erros de medidas ,bem como a suposição do creme utilizado ser uma substância homogênea e uma substância isotrópica.

\section{Agradecimentos}

A acadêmica, Mônica Ferreira de Sousa, agradece o suporte financeiro concedida pela Universidade Estadual de Goiás por meio do programa de bolsa de iniciação científica na modalidade $\mathrm{PBIC/UEG.}$

\section{Referências}

1. Tillmann, A. R.; Ghimarães, G.; Silva, S. M. M. L.; Determinação simultânea da condutividade térmica e da difusividade térmica variando com a temperatura. In: $15^{\circ}$ POSMEC - Simpósio do Programa de PósGraduação em Engenharia Mecânica, MG: FEMEC, 2005.

2. Özişik, M. N.; Transferência de calor. Rio de Janeiro: Guanabara Koogan, 1990.

3. Sweat, V. E. Experimental values of thermal conductivities of selected fruits and vegetables. Journal of Food Science, Chicago, n.39, p.1081-3, 1974.

4. Choi, Y.; Okos, M. R. The thermal properties of liquid foods-review. II.Paper N.83-6516, 1983b. In Proceedings of the Winter Meeting of the American Society of Agricultural Engineers. Chicago.

5. Ball, C. O. Olson, F. C. W. Sterilization in food technology. Theory, Practice and Calculation., Mc Graw Hill Book Co., New York, 1957.

6. Carciofi, B. A. M.; Faistel J.; Aragão G. M. F.; Laurindo, J. B. J. of Food Engineering, v.55, p. 89-94, 2002.

7. M. Markowski, I. Bialobrzewski, M. Cierachb, A. Paulo, J. B. J. of Food Engineering 65, 591-598, 2004.

8. Oro, A. C. P., Borges B. S., Igreja, G.,Moreira, M. F. P. Analise da Influencia da temperatura e da umidade na difusividade térmica efetiva da soja, In Anais do XVIII EAIC, Londrina-PR, 2009.

9. Incropera F. P. e Witt D. P.; Fundamentos da Transferência de Calor e de Massa; Rio de Janeiro; Guanabara Koogan, 1992.

10. E. Butkov, Física Matemática, Guanabara Dois, RJ, 1978.

11. Pinsky, M. A., Partial Differential Equations and Boundary-Value Problems with Applications, Mc. Graw-Hill Inc., New York, 1991, p. 174.

12. Churchill, R.V., Brown, J. W, Fouries Series and Boundary Value Problems, McGraw-Hill Book Company, New York, 1987.

\section{Mônica F. de Sousa, José S. Sales* \& Orlene S. da Costa}

Ciências Exatas e Tecnológicas. Universidade Estadual de Goiás, 75132-903, Anápolis (GO), Brasil

*E-mail: josé.sales@ueg.br 